\title{
Application of Compressed Sensing in NB-IoT-Based Structural Health Monitoring of Rail Tracks
}

\author{
Xin $\mathrm{Li}^{\mathrm{a}}$, Yan Yan ${ }^{\mathrm{b}, 1}$, Pan $\mathrm{Hu}^{\mathrm{a}}$, Qing Zhang, Haitao Wanga ${ }^{\mathrm{a}}$, Chao Wang ${ }^{\mathrm{c}}$, Yifan Chen ${ }^{\mathrm{d}}$, \\ Guiyun Tian ${ }^{\mathrm{b}}$ \\ ${ }^{a}$ College of Automation Engineering, Nanjing University of Aeronautics and \\ Astronautics, Nanjing, China \\ ${ }^{\mathrm{b}}$ School of Automation Engineering, University of Electronic Science and Technology \\ of China, Chengdu, China \\ ${ }^{\mathrm{c}}$ School of Electronic Science and Engineering, University of Electronic Science and \\ Technology of China, Chao Wang \\ ${ }^{\mathrm{d}}$ School of Life Science and Technology, University of Electronic Science and \\ Technology of China, Yifan Chen
}

\begin{abstract}
Ultrasonic guided-wave testing is one of the most widely used technology for Structural Health Monitoring (SHM) of rail tracks. Currently, cable is the main tool of signal transmission for guided wave-based track monitoring systems. The installation of cables can significantly increase the system cost and restrict the flexibility of system deployment. In recent years, the NB-IoT technology has been gradually applied to the field of SHM, it offers long-range wireless communication among a large-scale sensor networks at the cost of minimum construction and maintenance. One primary obstacle hindering the integration of NB-IoT and guided wave-based track monitoring system is that the limited channel bandwidth of NB-IoT leads to significant transmission delay when transmitting the ultrasonic guided-wave signal sampled at Nyquist rate. In this paper, a Compressed Sensing (CS) framework for NB-IoT based rail-track monitoring system is proposed. The proposed CS framework utilizes the sparsity feature of the ultrasonic lamb-wave signal to enable sub-Nyquist sampling and maintain the feature of the measured signal at a low compression rate. To validate the proposed CS framework, the propagation time of lamb-wave is selected as the performance metrics. The experimental results show that compared with the traditional sampling method, the propagation time of lamb wave in rail track can be accurately extracted when the sampling rate is set to $100 \mathrm{kHz}$, therefore, the channel bandwidth of NB-IoT can meet the delay-free data transmission of a single ultrasonic sensor.
\end{abstract}

Keywords. NB-IoT, compressed sensing, structural health monitoring, rail-track inspection

\section{Introduction}

The structural integrity of rail tracks has always been paid an extra attention by railway operators. When the track operates under high load for a long time, cracks are easy to appear at the rail tracks. Ultrasonic guided-wave testing has been widely used for Structural Health Monitoring (SHM) of rail tracks ${ }^{[1 \sim 2]}$ mainly due to its ability to inspect a long-range of continuously welded rail tracks from a single transducer location $^{[3]}$. Currently, the cable is the main tool of signal transmission for guided wave-based rail track monitoring systems. The installation of cables can significantly increase the system cost and restrict the flexibility of system deployment. In recent 
years, the NB-IoT technology has been gradually applied to the field of SHM, it offers long-range wireless communication among a large-scale sensor networks at the cost of minimum construction and maintenance. For example, $\mathrm{Hu}$ et $\mathrm{al}^{[4]}$. applied $\mathrm{ZigBee}$ technology to guide wave monitoring of rail. The integration of IoT and the ultrasonic guided-wave testing is expected to provide a promising solution for efficient, fast, and low-cost ultrasonic monitoring system ${ }^{[5]}$. Because Lamb Wave can detect both internal damage and surface damage, it is often used as an ultrasonic guided wave for structural damage detection. The frequency of the Lamb-wave signal generally ranges from $60 \mathrm{kHz}$ to $200 \mathrm{kHz}{ }^{[6]}$. To minimize the signal distortion, combining the Nyquist sampling theorem ${ }^{[7]}$ with engineering practice, the sampling frequency is usually set to ten times of the frequency of lamb-wave signal. Many wireless standards can be used in IoT scenarios, such as WiFi, Bluetooth, GSM, etc. Among these standards, the NB-IoT plays a leading role by providing long-distance and robust communication to large-scaled, small, low-cost, and battery-powered sensors. Compared to WiFi, Bluetooth, NB-IoT provides a longer communication distance. Compared to GSM, it allows the sensor to achieve the same communication distance while consuming less power. Therefore, NB-IoT is considered as a promising wireless technology for the application of structural health monitoring ${ }^{[8 \sim 9]}$. However, the channel bandwidth of NB-IoT is significantly limited, which ranges from $160 \mathrm{Kbps}$ to $250 \mathrm{Kbps}{ }^{[10]}$, leading to significant transmission delay when transmitting the ultrasonic guided-wave signal sampled at Nyquist rate ${ }^{[11]}$.

In recent years, a novel signal acquisition and compression theory named Compressed Sensing (CS) has been widely used in the application scenarios that require data compression. Unlike conventional data compression methods, CS allow the signal to be sampled at a sub-Nyquist rate, thereby reducing the amount of data to be transmitted, and then reconstructing the compressively sampled signal into a complete raw signal. Yuequan Bao et al. ${ }^{[12]}$ used the acceleration data collected by the SHM system of the Yellow River Road Bridge in Binzhou, Shandong to study the data compression capability of CS. T Di Ianni et al. ${ }^{[13]}$ studied the recovery of guided wave fields of aluminum plates and composite plates under different sparse substrates, and determined that sparse substrates were an important factor affecting the CS reconstruction performance. Yong Huang et al. ${ }^{[14]}$ proposed a Bayesian Compressive Sensing(BCS) algorithm suitable for civil structural health monitoring (SHM) system, which can achieve perfect lossless compression performance at a high compression ratio. Jayawardhana M. et al. ${ }^{[15]}$ studied SHM data acquisition based on CS, especially the application of CS in damage detection and location.

This paper proposes a CS framework for NB-IoT based rail-track monitoring system is proposed. The proposed CS framework utilizes the sparsity feature of the ultrasonic lamb-wave signal to enable sub-Nyquist sampling and maintain the feature of the measured signal at a low compression rate. The proposed CS framework is validated by a set of experiments for measuring the lamb-wave propagation time in a rail-track specimen. Corresponding results show that the minimum compression rate that can maintain the characteristic quantity of lamb wave propagation time is obtained. that under the condition of no loss of lamb wave signal characteristics, the compressed sensing algorithm based on wavelet sparse basis can reduce the sampling frequency of lamb wave signal in the ultrasonic structural health monitoring system for rail-tracks to $10 \%$ of the traditional sampling frequency, satisfying the communication requirements of NB-IoT. The remainder of this article is organized as follows: In section 2, the CS theory in the lamb wave monitoring system is briefly introduced, and how to select a 
sparse basis to maintain the eigenvalue of lamb and the workflow of CS technology are described. In the third part, a verification test is designed to verify the proposed CS framework, the fourth part evaluates the performance of the proposed CS framework under different compression rates and discusses the minimum compression rate that can maintain the characteristic quantity of lamb. Finally, conclusion and future work are given.

\section{Compressed sensing for lamb monitoring system}

\subsection{Basic Principle of Compressed Sensing}

The core idea of CS is that the original signal can be linearly measured by the measurement matrix during signal acquisition and a small number of measurements can be recorded. The nonlinear algorithm is used to recover the exact approximation of the original signal from a small number of measured value ${ }^{[16]}$.

An important premise of compressed sensing is that the signal can be represented by the linear combination of a set of non-zero elements in certain sparse basis. Suppose the expression of signal $\mathrm{x}(\mathrm{n})$ on the sparse basis $\Phi$ is:

$$
\mathbf{x}=\varphi \times \alpha, \text { or } \mathbf{x}=\sum_{\mathrm{i}=1}^{N} \varphi_{\mathrm{i}} \times \alpha_{\mathrm{i}}
$$

There are k non-zero elements in $\boldsymbol{\alpha}, \mathrm{k}<<\mathrm{N}$, then signal $\mathrm{x}(\mathrm{N})$ is $\mathrm{k}$ sparse on a sparse basis $\Phi$. Formula (1) is called sparse representation, that is, in a sparse domain, only $\mathrm{K}$ elements are useful, and other elements will not affect the original signal even if they are discarded. The sparsity of a time-domain signal could be found by performing some orthonormal transformation ${ }^{[17]}$.

The essence of compression is to project the signal $\mathrm{X}$ from the high dimensional space of $\mathrm{N} \times 1$ to the low dimensional space of $\mathrm{M} \times 1$ through the matrix $\phi$ independent of $\varphi$ and obtain the linear measurement value y of $\mathrm{M} \times 1$ to realize the signal compression sampling. Its mathematical process can be described as follows:

$$
\mathrm{y}=\phi \times \mathrm{x}
$$

By substituting formula (1) into formula (2), we can get:

$$
\mathrm{y}=\phi \times \varphi \times \alpha=\Theta \times \alpha
$$

Where, $\phi$ is an $\mathrm{M} \times \mathrm{N}$ matrix, called the measurement matrix, and $\Theta=\phi \times \varphi$ is an $\mathrm{M} \times \mathrm{N}$ matrix, called the observation matrix. Signal reconstruction means that $\mathrm{y}$ is known to invert $\mathrm{x}$ through formula (3). Due to the sparsity of $\mathrm{X}$, namely $\mathrm{M}<<\mathrm{N}$, which ensures that formula (3) has a solution, but also that formula (3) has an infinite number of solutions. There are many methods to find the optimal solution in formula (3), and 
this paper adopted Orthogonal Matching Pursuit (OMP) to reconstruct the lamb signals in ultrasonic guided wave-based structural health monitoring system for rail-tracks.

\subsection{Investigation of Sparse Representation of Lamb-wave Signal}

The sparsity of a signal in a sparse domain is the prerequisite for signal compression sampling. Signal sparsity also affects the accuracy of signal reconstruction. In general, the better the sparsity of the signal under a sparse basis, the higher the accuracy of the reconstructed signal after compression ${ }^{[18 \sim 19]}$.

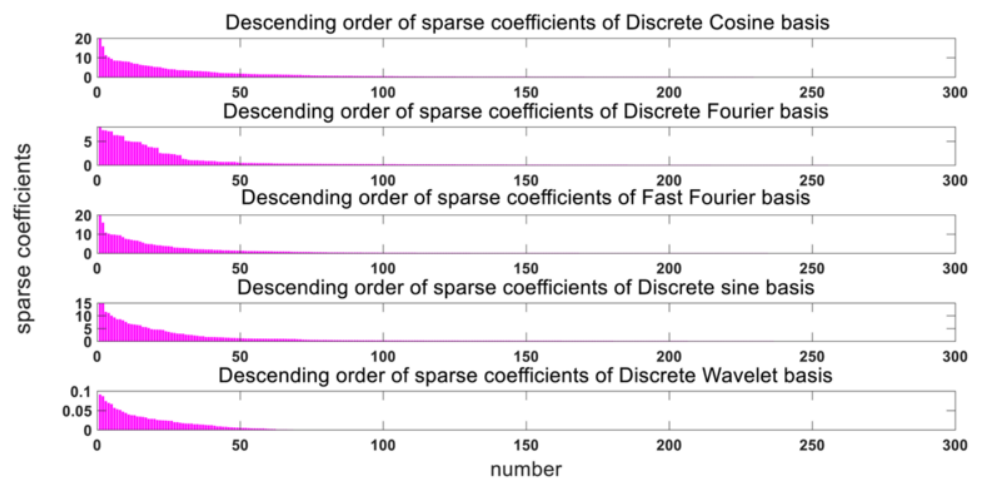

Figure 1. The sparse distribution of lamb signal in different sparse domains

To minimize the compression ratio, we investigate the sparse representation of the lamb-wave signal using various widely used sparse transform basis, which including Discrete Fourier Transform (DFT), Discrete Wavelet Transform (DWT), Discrete Cosine Transform (DCT), and Fast Fourier Transform (FFT), Discrete Sine Transform (DST). As shown in Figure 1, the sparse coefficients of lamb wave signals in the sparse domain such as DCT, DFT, DST, FFT, and DWT are compared. The sparsity coefficients refer to a set of non-zero elements of the signal in the sparse domain, it represents the sparseness of the signal. For a sparse transform basis, the fewer the non-zero element can be used to represent the signal, the higher the sparseness and the higher accuracy of the reconstructed signal ${ }^{[19]}$. As can be seen from Figure 1 , in the sparse domain such as DCT, DFT, DST and FFT, the number of sparse coefficients of lamb signal is greater than 140, and the number of sparse coefficients is the highest in the DST sparse domain. However, in the wavelet sparse domain, the number of sparse coefficients of lamb signal is 60 , and its sparsity is the best. The better the signal sparsity, the less the characteristic loss of reconstructed signal. In the ultrasonic rail structure health monitoring system, the flight time of $\mathrm{a} 0$ and $\mathrm{s} 0$ modal lamb waves is often used as an important characteristic quantity of structural damage ${ }^{[2]}$. In order to verify the above theory, the flight time of $\mathrm{a} 0$ and $\mathrm{s} 0$ modal lamb waves of reconstructed signals and original signals under different sparse bases is compared based on the peak time of the wave. The comparison results are shown in Table 1, in which the flight time of $\mathrm{a} 0$ and $\mathrm{s} 0$ modal lamb waves of reconstructed signals under wavelet sparse basis has the minimum error with the original signal. 
Table 1. Preservation of feature quantities of lamb under different sparse basis

\begin{tabular}{lcccc}
\hline $\begin{array}{l}\text { Sparse } \\
\text { matrix }\end{array}$ & $\begin{array}{c}\text { Peak arrival } \\
\text { time of } \\
\text { Lamb s0 } \\
\text { wave(us) }\end{array}$ & $\begin{array}{c}\text { the peak arrival time (us) of } \\
\text { the Lamb s0 wave extracted } \\
\text { from reconstructed signals }\end{array}$ & $\begin{array}{c}\text { Peak arrival } \\
\text { time of } \\
\text { Lamb a0 } \\
\text { wave(us) }\end{array}$ & $\begin{array}{c}\text { the peak arrival time } \\
\text { (us) of the Lamb a0 } \\
\text { wave extracted from } \\
\text { reconstructed signals }\end{array}$ \\
\hline $\mathrm{dwt}$ & 88.9 & 88.8 & 113.3 & 113.3 \\
$\mathrm{dft}$ & 88.9 & 88.6 & 113.3 & 113.3 \\
$\mathrm{dct}$ & 88.9 & 94.2 & 113.3 & 114.2 \\
$\mathrm{dst}$ & 88.9 & 89.2 & 113.3 & 112.8 \\
$\mathrm{fft}$ & 88.9 & 88.5 & 113.3 & 113.2 \\
\hline
\end{tabular}

\section{Experimental set-up and Performance Evaluation}

\subsection{Experimental set-up}

The peak arrival times of a0 and s0 modes of lamb-wave is often used as the characteristic quantity of damage detection in lamb wave monitoring. To evaluate the effect of the CS framework on lamb wave characteristics, a series of experiments were carried out. The raw signal is collected by the UT monitoring system shown in Figure 2 (a). Excitation and receiving sensors are shown in Figure 2 (b). The system consists of an excitation signal source, excitation piezoelectric sensor, receiving piezoelectric sensor, conditioning circuit, and oscilloscope responsible for signal acquisition. The specific experimental parameters are shown in Table 2. This system is aimed at the half - moon crack at bottom of the rail ${ }^{[20]}$, and the size of target damage is tens $\mathrm{mm}$ order ${ }^{[21]}$.

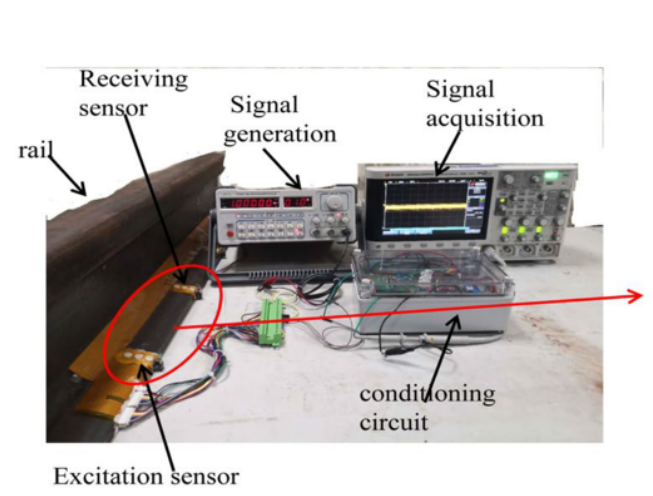

(a)

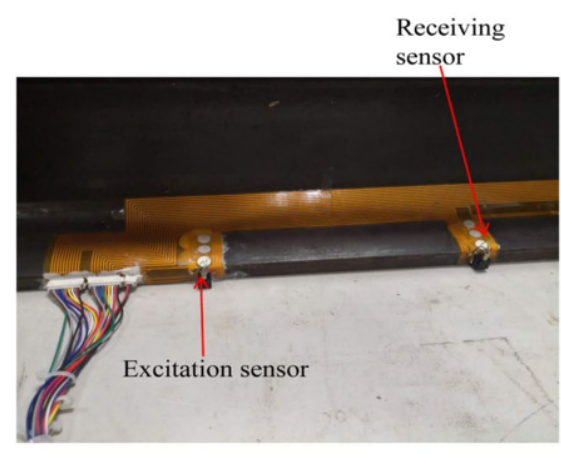

(b)

Figure 2. Lamb monitoring system for rail-track

Table 2. Experimental parameters

\begin{tabular}{cccc}
\hline $\begin{array}{c}\text { Monitoring } \\
\text { Distance }\end{array}$ & $\begin{array}{c}\text { Frequency of } \\
\text { lamb-wave signal }\end{array}$ & $\begin{array}{c}\text { Conditioning circuit } \\
\text { amplification factor }\end{array}$ & Sampling Frequency \\
\hline $200 \mathrm{~mm}$ & $140 \mathrm{KHz}$ & 300 & $1 \mathrm{MHz}$ \\
\hline
\end{tabular}




\subsection{The workflow of CS in ultrasonic guided wave-based structural monitoring system} for rail-tracks

As shown in Figure 3, the frame diagram of compressed sensing technology is proposed for this paper. In this framework, for lamb signals in ultrasonic-based structural health monitoring system for rail-tracks, the sparse coefficient of lamb signals in the wavelet sparse domain is obtained by using the wavelet sparse bases selected in Section 2.2. Then, Gaussian random matrix is adopted as the measurement matrix and the OMP algorithm is adopted to solve the signal reconstruction problem.

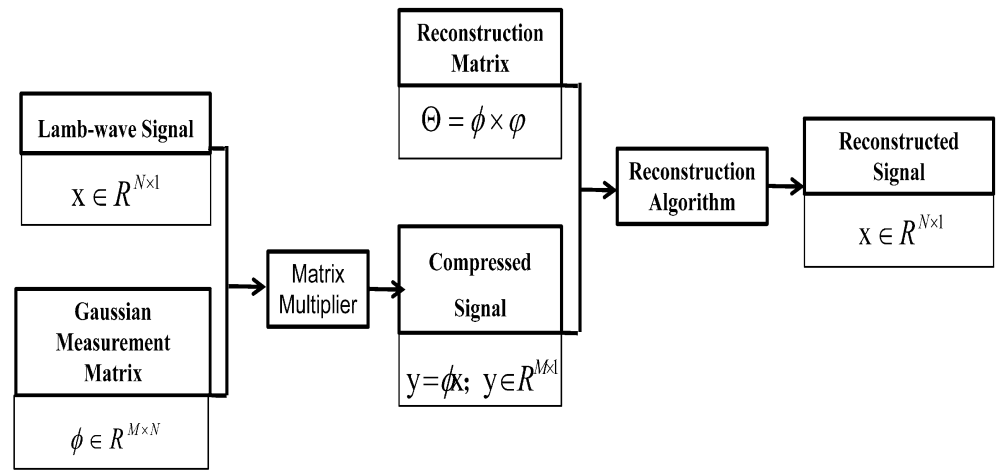

Figure 3. The workflow of CS in UT monitoring system for rail-tracks

\subsection{Performance Evaluation}

In the experiment, the raw signal sampled at $1 \mathrm{MHz}$ contains 2000 individual values, When the compression ratio was 0.3, 600 samples were measured for 2000 samples When comparing the raw signal and the compressive measured signal, it can be seen clearly that the peak arrival time of the two signals is almost the same, which means that the characteristic quantity of the original signal is maintained accurately in the recovered signal. Figure 4 depicts the raw signal sampled at $1 \mathrm{MHz}$, sparse domain, reconstructed signal, and compressive measured signal, respectively.

According to CS theory, according to the compression ratio theory, although low compression ratio is conducive to the collection and storage of data in the ultrasonic testing system of the Internet of Things, it is not conducive to the maintenance of lamb feature quantity. Figure 5 shows the evaluation of the reconstruction accuracy of the above CS framework under different sampling scores. It includes an absolute error, root mean square error, signal to noise ratio $(\mathrm{dB})$, and peak signal to noise ratio $(\mathrm{dB})$. The experimental results show that when the sampling fraction is greater than 0.1 , the proposed CS framework achieves better reconstruction performance and remains within a relatively stable range. 

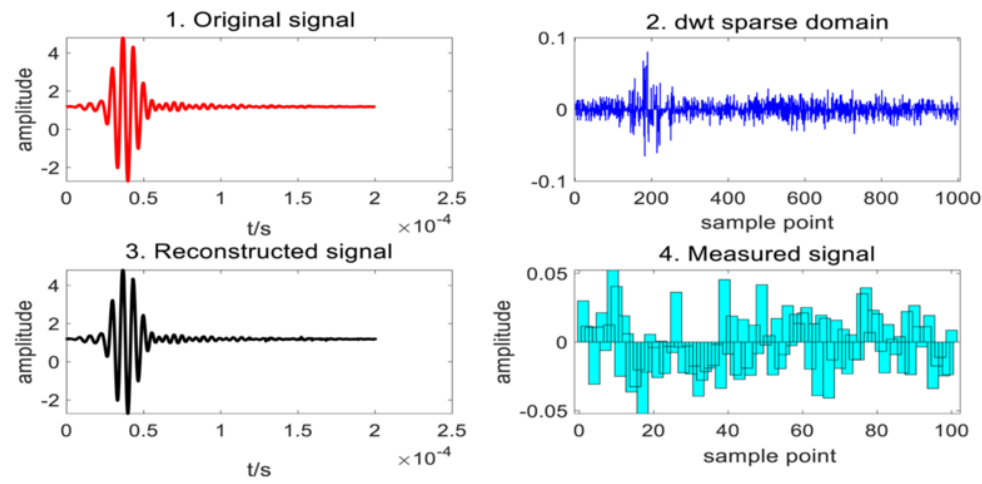

Figure 4. Performance of CS framework
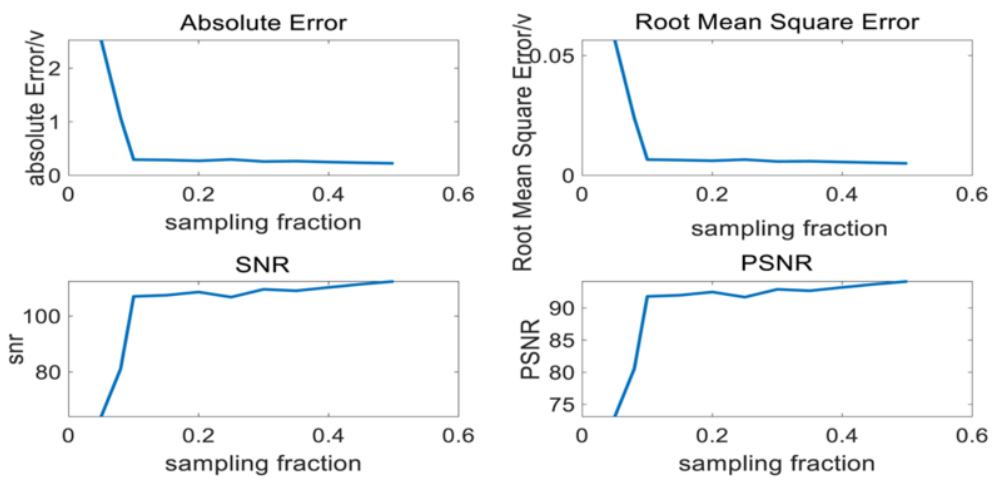

Figure 5. Performance Comparison of the CS method under different compression ratio

As described in Section 2.2, the flight time of the a 0 and s0 lamb waves is an important feature of the rail-based ultrasonic structural health monitoring system. This section discusses the lower bound of compression rate of CS algorithm based on wavelet sparse basis when the lamb feature loss is the smallest in ultrasonic structure health monitoring system for rail tracks. Figure 6 shows the original signal and the reconstructed signal when the compression ratios are set to $0.3,0.1,0.08$, and 0.05 respectively. Table 3 shows the peak arrival time of a0 mode and s0 mode of the original signal and the compression reconstruction signal under different compression rates. As shown in Table 3, when compression ratio $>0.1$, the reconstructed signal retains the basic characteristics of the original lamb signal when compression ratio < 0.1 , the reconstructed signal cannot maintain the basic characteristics of the original lamb signal, resulting in a large error. Therefore, when the lamb signal in the rail is compressed, the compression rate should not be lower than 0.1 . When the compression rate is set to 0.1 , the corresponding sampling rate is $100 \mathrm{kHz}$. The bandwidth of NB-IoT can be expressed as:

$$
\mathrm{W}=\mathrm{D} \times \mathrm{F} \times \mathrm{N}
$$

Where $\mathrm{W}$ refers to the bandwidth in bits per second, D stands for the sampling resolution (bits/sample), F represents the sampling frequency (sample/second), and $\mathrm{N}$ denotes the number of channels. Since the bandwidth of NB-IoT is $250 \mathrm{Kbps}$, and D $\geq 1$, 
it can be deduced that the upper bound of sampling frequency is $250 \mathrm{kHz}$ per channel. Given that the minimum sampling frequency is $100 \mathrm{kHz}$ when applying the proposed CS framework, therefore, the data generated by a single transducer can be transmitted using NB-IoT without delay.
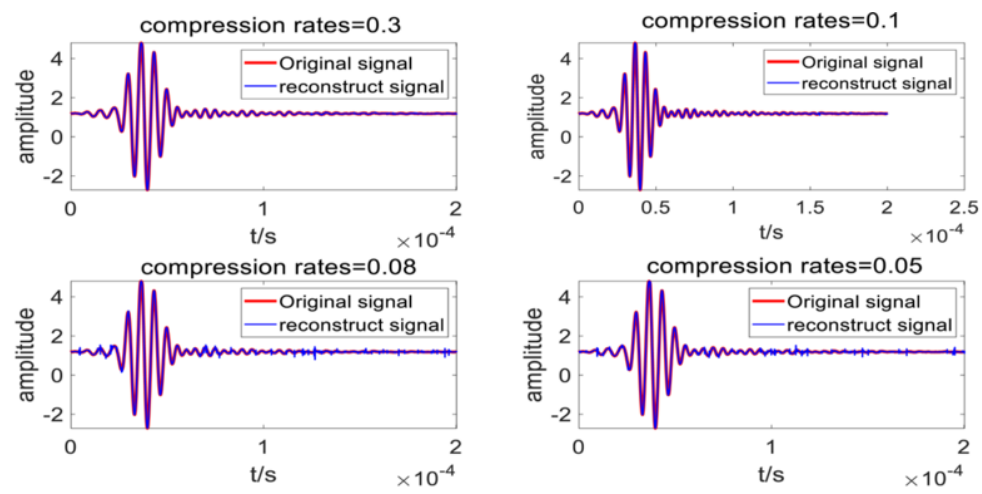

Figure 6. Original signal and reconstructed signal at different compression rates

Table 3. Peak arrival time of original signal and reconstructed signal under different compression rates

\begin{tabular}{lcccc}
\hline $\begin{array}{c}\text { Compress } \\
\text { rate }\end{array}$ & $\begin{array}{c}\text { Peak arrival } \\
\text { time of } \\
\text { Lamb s0 } \\
\text { wave (us) }\end{array}$ & $\begin{array}{c}\text { Peak arrival time of } \\
\text { the Lamb s0 wave (us) } \\
\text { extracted from } \\
\text { reconstructed signals }\end{array}$ & $\begin{array}{c}\text { Peak arrival time } \\
\text { of Lamb a0 wave } \\
\text { (us) }\end{array}$ & $\begin{array}{c}\text { Peak arrival time } \\
\text { (us) of the Lamb a0 } \\
\text { wave extracted from } \\
\text { reconstructed signals }\end{array}$ \\
\hline 0.3 & 88.9 & 88.8 & 113.3 & \\
0.1 & 88.9 & 88.8 & 113.3 & 113.3 \\
0.08 & 88.9 & 88.2 & 113.3 & 116.2 \\
0.05 & 88.9 & 92.9 & 113.3 & 117.1 \\
\hline
\end{tabular}

\section{Conclusion and future works}

This paper provides a theoretical and experimental basis for realizing the integration of NB-IoT and ultrasonic guided wave-based structure health monitoring system for rail tracks. The experimental results show that under the condition of no loss of lamb wave signal characteristics, the compressed sensing algorithm based on wavelet sparse basis can reduce the sampling frequency of lamb wave signal in the ultrasonic structural health monitoring system for rail-tracks to $10 \%$ of the traditional sampling frequency, satisfying the communication requirements of NB-IoT.

In future stage, on the one hand, it is necessary to further verify the influence of the compressed sensing framework proposed in this paper on the characteristics of lamb wave reflected by the damage; on the other hand, sparse dictionary with optimal sparsity estimation can be developed to further reduce the minimum sampling frequency, and a more efficient measurement matrix and reconstruction algorithm should be designed to achieve a lower compression rate on the premise of maintaining the signal characteristics of reconstructed lamb signal. 


\section{Acknowledgement}

This work was funded by the National Natural Science Foundation of China [grant numbers 6152780147], the Key Laboratory of Non-Destructive Testing and Monitoring Technology for High-Speed Transport Facilities of the Ministry of Industry and Information Technology.

\section{Reference}

[1] D. Barke, W.K. Chiu, Structural health monitoring in the railway industry: a review, Structural Health Monitoring 4 (2005), 81-93.

[2] J.L. Rose, M.J. Avioli, P. Mudge, et al. Guided wave inspection potential of defects in rail, $N d t \& E$ International 37 (2004), 153-161.

[3] P.W. Loveday, C.S. Long, Influence of resonant transducer variations on long range guided wave monitoring of rail track, AIP Conference Proceedings. AIP Publishing LLC 1706 (2016), 150004.

[4] P. $\mathrm{Hu}$, et al. Wireless localization of spalling in switch-rails with guided waves based on a time-frequency method, IEEE Sensors Journal 23 (2019), 11050-11062.

[5] A. Ahmed, Y. Kumar, Internet of things (IoT) platform for structure health monitoring, Wireless Communications and Mobile Computing 2017 (2017), 1-10.

[6] B.C. Lee, W.J. Staszewski, Modelling of lamb waves for damage detection in metallic structures: Part I. Wave propagation, Smart Materials and Structures 12 (2003), 804.

[7] Wikipedia. 2020. "Nyquist-Shannon sampling theorem" Last modified July 172020. https://en.wikipedia.org/wiki/Nyquist-Shannon sampling theorem

[8] Y. Yan, et al. A study on data loss compensation of WiFi-based wireless sensor networks for structural health monitoring, IEEE Sensors Journal 16 (2015), 3811-3818.

[9] C. Hemalatha, M.V. Rajkumar, M. Gayathri. IOT based building monitoring system using GSM technique, IOSR-Journal of Electronics and Communication Engineering 12 (2017), 68-75.

[10] R. Ratasuk, B. Vejlgaard, N. Mangalvedhe, et al. NB-IoT system for M2M communication, 2016 IEEE Wireless Communications and Networking Conference (2016), 1-5.

[11] A. Perelli, T. Di Ianni, A. Marzani, et al. Model-based compressive sensing for damage localization in lamb wave inspection, IEEE Transactions on Ultrasonics Ferroelectrics and Frequency Control 60 (2013), 2089-2097.

[12] Y. Bao, J.L. Beck, H. Li. Compressive sampling for accelerometer signals in structural health monitoring, Structural Health Monitoring 10 (2011), 235-246.

[13] T. Di Ianni, L. De Marchi, A. Perelli, et al. Compressive sensing of full wave field data for structural health monitoring applications, IEEE Transactions on Ultrasonics Ferroelectrics and Frequency Control 62 (2015), 1373-1383.

[14] Y. Huang, J.L. Beck, S. Wu, et al. Robust bayesian compressive sensing for signals in structural health monitoring, Computer-Aided Civil and Infrastructure Engineering 29 (2014), 160-179.

[15] M. Jayawardhana, X. Zhu, R. Liyanapathirana, et al. Compressive sensing for efficient health monitoring and effective damage detection of structures, Mechanical Systems and Signal Processing 84 (2017), 414-430.

[16] D.L. Donoho, Compressed sensing, IEEE Transactions on Information theory 52 (2006), 1289-1306

[17] M. Rani, S.B. Dhok, R.B. Deshmukh, A systematic review of compressive sensing: Concepts, implementations and applications, IEEE Access 6 (2018), 4875-4894.

[18] I. rović, V. Papić, C. Ioana, et al. Compressive sensing in signal processing: algorithms and transform domain formulations, Mathematical Problems in Engineering (2016), 1-16.

[19] Z. Zhang, Y. Xu, J. Yang, et al. A survey of sparse representation: algorithms and applications, IEEE Access 3 (2015), 490-530.

[20] D.F. Cannon, K.O. Edel, S.L. Grassie, et al. Rail defects: an overview, Fatigue \& Fracture of Engineering Materials \& Structures 26 (2003), 865-886.

[21] C. Ramadas, M. Janardhan Padiyar, K. Balasubramaniam et al. Delamination size detection using time of flight of anti-symmetric (A0) and mode converted A0 mode of guided Lamb waves, Journal of Intelligent Material Systems and Structures 21 (2010), 817-825. 